\title{
Multi-omics differentially classify disease state and treatment outcome in pediatric Crohn's disease
}

Gavin M. Douglas', Richard Hansen², Casey M. A. Jones ${ }^{3}$, Katherine A. Dunn ${ }^{4}$, André M. Comeau ${ }^{5}$, Joseph P. Bielawski ${ }^{4}$, Rachel Tayler², Emad M. El-Omar ${ }^{6}$, Richard K. Russell ${ }^{2}$, Georgina L. Hold ${ }^{6}$, Morgan G. I. Langille ${ }^{1,3,5^{*}}$ and Johan Van Limbergen ${ }^{7}$

\begin{abstract}
Background: Crohn's disease (CD) has an unclear etiology, but there is growing evidence of a direct link with a dysbiotic microbiome. Many gut microbes have previously been associated with $C D$, but these have mainly been confounded with patients' ongoing treatments. Additionally, most analyses of CD patients' microbiomes have focused on microbes in stool samples, which yield different insights than profiling biopsy samples.

Results: We sequenced the $16 S$ rRNA gene (16S) and carried out shotgun metagenomics (MGS) from the intestinal biopsies of 20 treatment-naive $C D$ and 20 control pediatric patients. We identified the abundances of microbial taxa and inferred functional categories within each dataset. We also identified known human genetic variants from the MGS data. We then used a machine learning approach to determine the classification accuracy when these datasets, collapsed to different hierarchical groupings, were used independently to classify patients by disease state and by CD patients' response to treatment. We found that 16S-identified microbes could classify patients with higher accuracy in both cases. Based on follow-ups with these patients, we identified which microbes and functions were best for predicting disease state and response to treatment, including several previously identified markers. By combining the top features from all significant models into a single model, we could compare the relative importance of these predictive features. We found that 16S-identified microbes are the best predictors of CD state whereas MGS-identified markers perform best for classifying treatment response.
\end{abstract}

Conclusions: We demonstrate for the first time that useful predictors of CD treatment response can be produced from shotgun MGS sequencing of biopsy samples despite the complications related to large proportions of host DNA. The top predictive features that we identified in this study could be useful for building an improved classifier for $C D$ and treatment response based on sufferers' microbiome in the future.

The BISCUIT project is funded by a Clinical Academic Fellowship from the Chief Scientist Office (Scotland)—CAF/08/01.

Keywords: Crohn's disease, Treatment response, Machine learning, Microbiome, Treatment-naïve, Pediatric

\footnotetext{
* Correspondence: morgan.g.i.langille@dal.ca

Gavin M. Douglas and Richard Hansen are joint first authors

Georgina L. Hold, Morgan G. I. Langille, and Johan Van Limbergen are joint

senior authors

'Department of Microbiology and Immunology, Dalhousie University, Halifax,

NS, Canada

${ }^{3}$ Department of Pharmacology, Dalhousie University, Halifax, NS, Canada

Full list of author information is available at the end of the article
} 


\section{Background}

Crohn's disease (CD) is an inflammatory bowel disease (IBD) classically characterized by abdominal pain, rectal bleeding and weight loss. Recurring flares of IBD cause lifelong, far-reaching consequences for patients that can affect lifestyle and overall health $[1,2]$. CD differs from the other form of IBD-ulcerative colitis-in that CD can affect any part of the gastrointestinal tract, can be discontinuous, and can involve granulomatous inflammation [3]. There is a growing need to understand the etiology of $C D$ due to the worldwide increase in annual incidence [4], particularly in children [5].

Although the etiology of $\mathrm{CD}$ is unclear [6], there is growing evidence for the dysbiosis hypothesis. This model postulates that a shift in the balance between commensal and pathogenic intestinal microbes interacting with the host's immune system contributes to CD onset. In support of this model, large-scale differences in bacterial abundances have long been associated with $C D$ [7]. The most reproducible finding has been a decrease in alpha-diversity in $\mathrm{CD}$ patients compared to controls [8-10]. Several particular changes in taxonomic abundances have been linked to this dysbiotic state; for instance, Firmicutes tend to be at lower proportion and Gammaproteobacteria at higher proportion in CD patients [11]. Most taxonomic profiles of CD patients have been based on stool samples, which yield drastically different insights into CD pathogenesis when compared with mucosal washing of the mucosal-luminal interface (MLI) and intestinal biopsy samples [8]. Irrespective of body site, it is unclear whether these shifts in microbiota are a cause or a symptom of the disease. However, there is reason to believe that the microbiome contributes to CD etiology due to several observations. Firstly, children that are exposed to antibiotics in the first year of life are more likely to develop IBD [12], which could be related to acquiring a dysbiotic state. Also, many CD risk loci are linked to pattern recognition receptors (PRRs) and cytokines that regulate the host immune system [13].

PRRs generate responses against pathogenic bacteria while identifying commensal bacteria within the human microbiome. The best-known example of a PRR linked to $\mathrm{CD}$ is the nucleotide-binding oligomerization domain containing protein 2 (NOD2) gene that codes for an intracellular PRR. Loss of function mutations in the gene lead to increased inflammation due to impaired clearance of intestinal bacteria that are harmful to the gut [14]. Despite these reproducible links to CD, risk mutations account for $<14 \%$ of disease variance across patients [15]. However, the concordance rate of CD between monozygotic twins ranges from 20 to $50 \%$, which is higher than several other complex diseases [16]. Nonetheless, risk loci alone do not explain $\mathrm{CD}$ onset and the relative importance of the microbiome in the onset of this disease is not well understood.

Here, we compare the relative importance of genetic risk loci and microbiota identified from intestinal biopsy samples for classifying treatment-naïve pediatric patients by disease state. We also demonstrate that CD patients' treatment response status can be classified by microbial features with high accuracy. Taxonomic and functional profiles discussed in this study are based on both $16 \mathrm{~S}$ sequencing and metagenomics (MGS) sequencing of the same intestinal biopsy samples. To our knowledge, this is the first report of shotgun MGS of CD intestinal biopsy samples.

\section{Methods}

\section{Sequenced samples}

Intestinal biopsies were previously taken from 20 Crohn's disease (CD) and 20 normal colon control patients as part of the "Bacteria in Inflammatory bowel disease in Scottish Children Undergoing Investigation before Treatment" (BISCUIT) cohort $[9,17]$. We did not perform a power test to predict what effect sizes could be detected with this sample size, but instead chose this sample size due to sequencing cost constraints. These patients were all under 17 years old with a mean age of 12.7 years. CD biopsies were obtained at the diagnostic endoscopy prior to commencing any therapy. We based CD diagnosis on the Paris Classification [18]. None of these patients used systemic antibiotics or steroids in the 3 months prior to their colonoscopy or immunosuppression at any point. Treatment response was classified as sustained remission following induction treatment response and was defined by physician global assessment and the requirement for treatment escalation (repeat induction therapy) before 24 weeks.

\section{Metagenomics sequencing and bioinformatic pipeline} Shotgun MGS preparation and sequencing was conducted by Génome-Québec (McGill University, Montréal, Québec) on an Illumina HiSeq. A mean of 110 million PE 100 base pair (bp) MGS reads were produced with a range of 72.7-135 million reads over all samples. We first concatenated FASTQ files containing forward and reverse reads into a single FASTQ per sample. We then screened out contaminant sequences by mapping all reads against the human (hg19) and PhiX (RTA) genomes using bowtie2 [19] (v2.2.6), which resulted in a mean of $90 \%$ of reads being excluded. This high percentage of contaminant reads is mainly due to the high proportion of human cells in biopsy samples, which is less of an issue for microbiome studies that focus on stool samples. After screening out these nonmicrobial reads, we classified the remaining reads taxonomically using MetaPhlAn2 [20] (v2.2.0) with the 
"-very-sensitive" global alignment option and into KEGG orthologs (KOs) using HUMAnN2 [21] (v0.11.1; http://huttenhower.sph.harvard.edu/humann2). Importantly, we found that running bowtie 2 in local alignment mode with MetaPhlAn2 resulted in many spurious hits, which were mainly represented by viruses. These taxa were not identified when global alignment was performed. We ran MUSiCC [22] (v1.0.2) to normalize the $\mathrm{KO}$ abundances within each sample by the median universal single-copy gene abundance, which controls for inter-sample variation in microbial genome sizes. We then ran HUMAnN2 on these normalized values to reconstruct KEGG module and pathway abundances within each sample. No taxa or functions were identified in the MGS of two samples, S34 and S38 (16S sequencing also failed for these samples, see below), which were excluded from downstream microbiome analyses.

\section{Calling human variants}

Due to the large percentage of human DNA in our MGS (see above), we were also able to call human variants from the same dataset. Although we used 133 loci for calculating the genetic risk score (see below), we called genome-wide variants to improve imputation accuracy in cases where samples were missing data at these sites. We began by mapping all MGS reads to the human genome (hg19) using the Burrows-Wheeler Alignment Tool's [23] (v0.7.12) mem algorithm, which resulted in a 98\% mapping rate. This mapping rate is higher than the rate for the metagenomic microbial pipeline due to the different algorithms used for each workflow. We then followed the Genome Analysis ToolKit's (GATK) [24] Best Practices workflow $[25,26]$ for variant calling. Pre-processing steps included marking duplicate reads, recalibrating base quality scores based on a model trained on known variants, and re-aligning reads around known insertions and deletions. We then ran the GATK (v3.5) program HaplotypeCaller to call variants using default parameters and variant quality score recalibration per the Best Practices workflow. These steps resulted in $16,333,869$ raw variants based on a genome-wide mean coverage of 7.5 reads across all 40 individuals. Due to the low genome-wide coverage, we also discarded variants based on several hard filters implemented by VCFtools [27] (v0.1.13): any variant not in Hardy-Weinberg equilibrium (cut-off significance of $P<1 \times 10^{-4}$ ), any variant called by $<6$ reads, or any variant with $>50 \%$ missing data. We retained 7,604,626 variants following these hard cut-offs. The 133 known risk loci were not required to pass these hard cut-offs.

\section{Imputing missing genotypes}

After calling variants genome-wide, we next imputed the missing genotypes for the 133 known $C D$ risk loci.
Three variants (rs9264942, rs11209026, rs6927022) were missing genotype calls in all samples and were excluded. Haplotype phasing and the first pass of imputation were performed with SHAPEIT [28] (v2.r837). IMPUTE2 [29, 30] (v2.3.2) was then run on SHAPEIT's phased output to impute the final genotypes. The HapMap phase II b37 genetic map was used for both imputation steps, and the 1000 Genomes Phase 3 [31] phased haplotypes were used as reference haplotypes. Default parameters were used for running both SHAPEIT and IMPUTE2.

\section{Genetic risk scores}

A custom Perl script was used to parse the IMPUTE2 output into a variant call format, and then PLINK [32] (v1.90b3.29) was used to convert this table into PED and MAP files. Per-sample genetic risk scores (GRS) were calculated using the Mangrove $\mathrm{R}$ package [33]. To calculate the GRS, we used the genotypes at these imputed risk loci, odds-ratio information for risk alleles, and minor allele frequencies from previously published genome-wide association studies $[15,34]$. We assumed a CD prevalence of $1 \%$ when calculating GRS ( $K$ value $=0.01)$.

\section{$16 \mathrm{~S}$ rRNA gene sequencing}

The intestinal biopsy samples were prepared for $16 \mathrm{~S}$ sequencing using our Microbiome Amplicon Sequencing Workflow [35]. Briefly, the pre-extracted DNA [17] was first amplified in duplicate using dual-indexing Illumina primers (forward: ACGCGHNRAACCTTACC; reverse: ACGGGCRGTGWGTRCAA) that targeted the V6-V8 region (438 bp) of the bacterial $16 \mathrm{~S}$ rRNA gene. The pooled duplicate PCR products were verified using highthroughput E-gels (Invitrogen), then purified and normalized using the SequalPrep 96-well Plate Kit (Invitrogen). Following quantification, the pooled samples were run on an Illumina MiSeq using PE $300+$ 300 bp v3 chemistry at the Integrated Microbiome Resource (Dalhousie University, Halifax, Nova Scotia).

\section{S rRNA gene bioinformatic pipeline}

We followed the Microbiome Helper standard operating procedure [35] to process the 16S rRNA gene data. Two CD samples (S34 and S38) were excluded from this pipeline due to low DNA quality and repeated sequencing failures, which left a total of 38 samples remaining (20 $\mathrm{CN}$ and $18 \mathrm{CD}$ ). A mean of 21,793 raw PE read pairs were produced over these remaining samples $(\min =9503 ; \max =40,392)$. Forward and reverse reads were then stitched together using PEAR [36] (v0.9.6) with an assembly rate $>80 \%$ for all samples except for sample S22 (68.7\% of reads assembled). We then filtered out stitched reads with a quality score $<30$ over $90 \%$ of bases using the FASTX toolkit (v0.0.14; http://hannonlab.cshl.edu/fastx_toolkit/). We also filtered 
out reads $<400 \mathrm{bp}$ or that did not have exact matches to the forward and reverse primers using BBMap (v35.82; https://sourceforge.net/projects/bbmap/). An average of $18.7 \%$ of the assembled reads per sample was discarded by these filters. Next, we removed chimeric sequences using UCHIME [37] (v6.1) with the parameters mindiv $=1.5$ and $\operatorname{minh}=0.2$, which resulted in an average of $16.3 \%$ of the assembled reads being discarded. Following these filters, a mean of 13,815 reads were remaining per sample $(\min =4427 ; \max =27,472)$. We ran open-reference $97 \%$ OTU picking using QIIME (v1.9.0) wrapper scripts with these filtered reads. Reference OTU picking was run against the Greengenes [38] (v13_8) database using SortMeRNA [39] (v2.0-dev, 29/11/2014) with a minimum query coverage of $80 \%$ and de novo OTU picking using SUMACLUST (v1.0.00; https://git.metabarcoding.org/ obitools/sumaclust/wikis/home/). We filtered out OTUs that were called by $<0.1 \%$ of reads and then rarefied read counts to 4000 reads per sample, which resulted in a final set of 984 OTUs. PICRUSt [40] (v1.0.0) was used to predict KEGG ortholog and pathway abundances based on reference OTU abundances. We compared the rarefied OTU abundances to non-rarified abundances after performing a centered log-ratio transformation [41]. Read counts were imputed with the count zero multiplicative method in the zCompositions $\mathrm{R}$ package [42] (v1.1.1) before performing the centered log-ratio transformation. We compared these workflows by evaluating how well models performed using abundance tables produced by each workflow. To evaluate concordance between MGS and 16S-identified genera, we calculated the Spearman's correlation $(\rho)$ of the relative abundances of $16 \mathrm{~S}$ genera at greater than $10 \%$ frequency and identified in both datasets.

\section{RISK validation cohort}

We downloaded single-end sequencing of the V4 region of the $16 \mathrm{~S}$ gene produced for the "Risk Stratification and Identification of Immunogenetic and Microbial Markers of Rapid Disease Progression in Children with Crohn's Disease" (RISK) cohort [8] from the National Center for Biotechnology Information under study accession PRJEB13679. We reduced this data to 773 biopsy samples that were either controls or $\mathrm{CD}$ patients and $\leq 18$ years old. To process this data, we first merged together sequencing replicates for the same samples. We then trimmed all reads to 130 nucleotides using Trimmomatic [43] (v0.36). The remaining steps were the same as the $16 \mathrm{~S}$ processing pipeline described above. The OTU table was rarefied to 4000 reads (42 samples with depth below this cut-off were discarded), which resulted in 2564 OTUs being called over 731 samples.

\section{Random forest classification}

For each dataset, we ran random forest (RF) models to classify disease state and treatment response separately. Each dataset was pre-processed, so only features with $>10 \%$ non-zero values were retained. Each table was then standardized by sample (subtracted the sample's mean and then divided by the sample's standard deviation). We ran RF models using the random forest [44] (v4.6.12) R package with default mtry values and used 712 as the random seed. All models were run with 10,001 trees except for the KO models which were run with 501 trees to reduce running time. RF model significance was determined by the permutation test implemented in the rfUtilities [45] (v2.0.0) R package. This test involves building a null distribution of out-of-bag (OOB) errors from RF models with randomized classes (e.g., the disease state column of the input table was randomized). Model significance is then determined by calculating whether $<5 \%$ of random permutation models have an $\mathrm{OOB}$ error less than or equal to the observed OOB error. Significance of RF models as tested by the above permutation procedure was treated as an omnibus test for any association between the signal derived from genetic data and the feature labels of each sample. This allowed us to identify at what level (e.g., family, genus and species) further investigation was warranted and supported our investigation of variable importance in some "datasets" and not others. Note that RF models make no assumptions about how the input features are distributed. Leave-one-out cross-validation was also run on each dataset to output accuracy for each model with the R package caret [46] (v6.0.77).

\section{Results}

Identifying CD-related SNPs, microbial taxa, and functions from intestinal biopsy samples

To investigate which microbial and genetic features best classify pediatric $\mathrm{CD}$ patients by disease state and treatment response, we sequenced the intestinal microbiomes of $20 \mathrm{CD}$ and 20 normal colon controls prior to any treatments. Both MGS and 16S sequencing were performed on the same biopsy samples. Much of the MGS data was comprised of human DNA (90\%), which was separated from the microbial DNA and used to call human genotypes. We combined the human genotypes at 133 known CD risk loci with known odds-ratios and allele frequencies to calculate a genetic risk score [33] (GRS) per sample. We then used the remaining microbial MGS reads, a mean of 10.7 million paired-end (PE) reads per sample, to call 115 independent taxa (summarized at the class level in Additional file 1: Figure S1). All microbial MGS reads were also used to identify the relative abundances of Kyoto Encyclopedia of Genes and Genomes [47] (KEGG) orthologs, pathways, and modules within each sample. Similarly, after filtering the $16 \mathrm{~S}$ 
amplicon reads, we retained an average of 13,815 stitched reads per sample. We performed open-reference clustering to call 984 operational taxonomic units (OTUs; summarized at the class level in Additional file 1: Figure S2). Overall, the relative abundances of MGS and 16S-identified genera were similar within the same biopsy samples (mean Spearman's $\rho=0.51$, standard deviation $=0.18$ ). Since sequencing read counts are a form of compositional data, we tested whether a centered log-ratio transform of the non-rarefied read counts [41] would result in improved model performance compared to rarefaction of all samples. Although the compositional-based methods performed slightly better for some feature tables, in the majority of cases, this transformation resulted in less accurate classification of patients (see below; Additional file 1: Figure S3), and so, we focused on the rarefied datasets for our analyses. We used these OTUs to infer the relative abundances of KEGG orthologs and pathways within each sample (see Additional file 2 for sample sequencing coverage and metadata). Two of the CD patients' microbial profiles were discarded due to low $16 \mathrm{~S}$ and MGS sequencing depth. These different datasets are outlined in Fig. 1 (see Additional file 1: Table S1 for sample details).

We then replicated two well-known predictors of CD: increased GRS [1] and a reduction in microbial alpha- diversity as proof of principle. We chose the simplest measure of alpha-diversity: the observed number of OTUs per sample (\# OTUs). Both GRS (one-tailed MannWhitney-Wilcoxon (M-W-W) test, $W=288, P=0.00837)$ and \# OTUs (one-tailed M-W-W test, $W=261.5$, $P=0.00894$ ) significantly differed between patients based on disease state in the expected directions (Additional file 1: Figure S4). To make these known predictors comparable to classification accuracies using datasets containing multiple features, we used an analogous method to calculate accuracy. Importantly, these metrics produced only marginal accuracies when used to classify patients by disease state (GRS, $62.5 \%$; \# OTUs, $71.1 \%$ ).

\section{Classifying samples by disease state}

We next investigated how well microbial datasets classify CD disease state. MGS and $16 \mathrm{~S}$ taxonomic datasets included strain and OTU-level-relative abundances respectively and were also collapsed at each level from species to phylum (Fig. 1). Functional datasets included KEGG ortholog and pathway counts for both sequencing technologies, as well as KEGG modules for MGS samples. In total, 19 datasets were entered as classifiers for disease state after standardization (each mean-centered and scaled by the standard deviation for each sample). We ran independent random forest (RF) models to

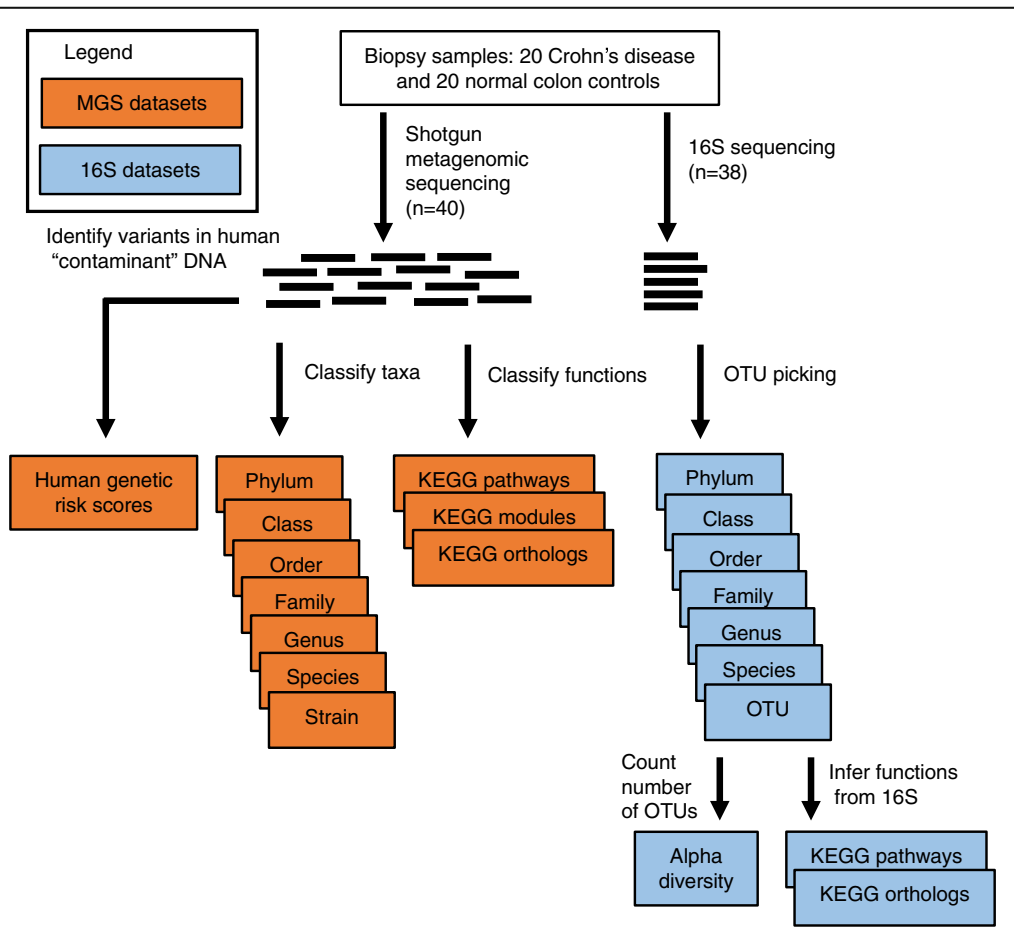

Fig. 1 Diagram of the different datasets used for classification in this study. Datasets in orange were derived from the shotgun metagenomic sequencing (MGS) data $(n=40)$ and the datasets in blue were derived from the $16 \mathrm{~S}$ rRNA gene (16S) sequencing data $\left(n=38^{*}\right)$. These datasets were used to classify both disease state and treatment response as input to random forest machine learning models. *Note two Crohn's disease samples were removed from both the 165 sequencing and MGS datasets due to low sequencing coverage, but their genetic profile was inferred from the MGS 
determine each dataset's classification accuracy (Fig. 2a; see Additional file 3). Each of the $16 \mathrm{~S}$ taxonomic datasets, except for the OTU level, could classify patients by disease state with high accuracy (maximum accuracy of $84.2 \%$ and $P<0.001$ based on genus level). The MGS strain, genus, family, and phylum taxonomic datasets also classified patients, but with lower accuracy than the $16 \mathrm{~S}$ datasets (maximum accuracy of $68.4 \%$ and $P=0.016$ based on strain level). The predicted $\mathrm{KO}$ abundances based on the 16S data and the MGS-identified KEGG modules both significantly classified patients as well (accuracies of 68.4 and $65.8 \%$ respectively).

One advantage of RF models is that they output variable importance metrics for each feature used in a model. We considered each RF model to be an omnibus test for each dataset, which enabled us to look at the ranking of variable importance in significant models to identify important features (see Additional file 4). Based on these metrics, the three most informative $16 \mathrm{~S}$ genera were Desulfovibrio, Akkermansia, and Butyricimonas (Additional file 1: Figure S5), whereas the top MGS genera were Alistipes, Oscillibacter, and Dorea. These top genera could differ since both top 16S genera were close to the detection limit threshold of the MGS data; they were only identified in a small number of samples (Additional file 1: Figure S6). Nonetheless, Akkermansia was ranked fourth in the MGS genus model despite being missed in several samples. The top features in the MGS strain model were strains of Alistipes putredinis, Clostridium symbiosum, and Faecalibacterium prausnit$z i i$. The 16S-inferred KOs, and the MGS modules were the only functional datasets that significantly classified samples by disease state (accuracy $=68.4 \%, P=0.043$ and accuracy $=65.8 \%, P=0.03$, respectively). The three top $16 \mathrm{~S}$ KOs were (1) K03785, which is involved in amino acid biosynthesis, (2) K09013, an Fe-S cluster assembly ATP-binding protein, and (3) K03809, a tryptophan repressor binding protein. The three top MGS modules were (1) M00144, NADH: quinone oxidoreductase, (2) M00362, nucleotide sugar biosynthesis, and (3) M00239, peptides/nickel transport system. Importantly, the datasets collapsed to different taxonomic and functional levels were not independent from each other, which is reflected by the fact that the top features in each taxonomic dataset tended to be part of the same lineage (e.g., the ranks above Desulfovibrio and Akkermansia were also top hits).

\section{Classifying samples by treatment response}

Next, we used these same 19 microbial datasets, after excluding normal colon control patients, to classify the $\mathrm{CD}$ patients as responders (RS) and non-responders (NR) to induction of remission treatments, started at the time of diagnosis (Fig. 2b; see Additional file 3). Clinical $\mathrm{CD}$ phenotypes were heterogeneous, but all included active colonic disease at the sampled location. Treatments were similarly not consistent across all patients, reflecting heterogeneity of phenotype, but instead were different combinations of exclusive enteral nutrition (EEN) therapy and immunosuppressive medications, as such representing 'real-world' CD treatment: 11 patients were on EEN, 3 were on prednisolone and EEN therapy, 4 were on mesalazine alone, and 2 were on prednisolone alone, as decided by their gastroenterologist at the time of diagnosis. Sustained response or non-response was defined as need for a second induction within 150 days of diagnosis or not (Additional file 1: Table S2). After classifying $\mathrm{CD}$ patients based on their response to
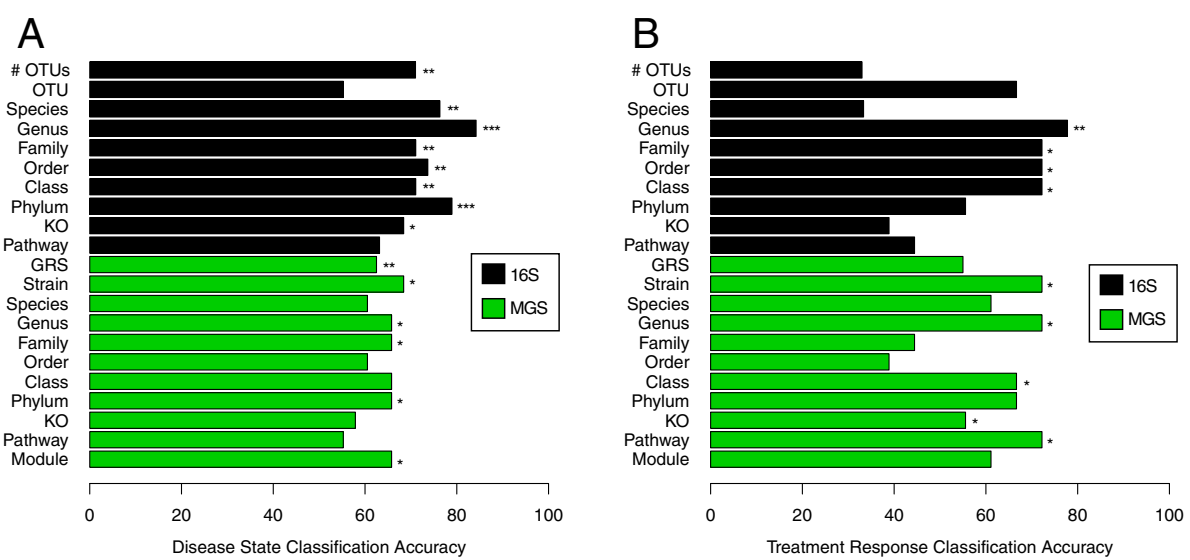

Fig. 2 Classification accuracies for all datasets classifying a disease state and $\mathbf{b}$ treatment response. Each bar corresponds to a different model. Accuracies are based on random forest (RF) leave-one-out cross-validation (LOOCV) in all cases, except for number of observed OTUs (\# OTUs) and genetic risk scores (GRS) which are based on LOOCV of simple linear cut-off models. The symbols *, **, and *** indicate significance at $P<0.05, P<0.01$, and $P<0.001$, respectively. RF model significances were based on a permutation test. $P$ values for \# OTUs and GRS are based on one-tailed Mann-WhitneyWilcoxon Tests 
induction treatment, $16 \mathrm{~S}$ genera were again the top dataset (accuracy $=77.8 \% ; P=0.008$ ). However, the MGS strain $(P=0.029)$, genus $(P=0.013)$, and KEGG pathway $(P=0.018)$ datasets could also classify patients with only slightly lower accuracy (accuracy $=72.2 \%$ for all three). We also found that alpha-diversity and GRS did not significantly differ between RS and NR patients (Additional file 1: Figure S7).

Using the same omnibus test approach as above, we were again able to identify the most informative features in each significant dataset (see Additional file 5). The top $16 \mathrm{~S}$ genera were Dialister, Bilophila, and Aggregatibacter in this analysis. The top MGS strains were subtypes of Parabacteroides merdae, Sutterella wadsworthensis, and an unclassified strain within the Lachnospiraceae family. The top MGS genera included Parabacteroides, Bacteroides, and an unclassified genus of Lachnospiraceae. The top MGS KEGG pathways included (1) ko00633, nitrotoluene degradation; (2) ko00250, alanine, aspartate, and glutamate metabolism; and (3) ko00230, purine metabolism. The top KOs were (1) K02954, a ribosomal protein, (2) K07259, which is involved in peptidoglycan biosynthesis, and (3) K07793, a putative tricarboxylic transport membrane protein.

\section{Comparing the relative importance of top features}

Although comparing RF model accuracies allows individual datasets to be evaluated, it does not allow the relative importance of features across datasets to be evaluated. To this end, we next compared the relative importance of the overall top features by running RF models using the top three features from the significant datasets for both $\mathrm{CD}$ state (Fig. 3a) and treatment response (Fig. 3b). The combined model for disease state classification performed with high accuracy (accuracy $=78.9 \%, P<0.001$ ), but notably, this was lower than the $16 \mathrm{~S}$ genera alone. In contrast, the combined model for treatment response classification performed better than the independent datasets (accuracy $=94.4 \%, P<0.001)$. As expected, many of these features in both models are highly correlated (Additional file 1: Figure S8 and S9); nonetheless, this approach yielded several useful results. Firstly, Akkermansia muciniphila was ranked as the most important feature for classifying disease state, followed by Verrucomicrobia and Verrucomicrobiales, which represent the phylum and order of $A$. muciniphila respectively. Number of OTUs was ranked fourth among these features, whereas GRS and other MGSderived features were ranked lower. Notably, 29/37 (78\%) of the microbial features in this model were at lower relative abundances in $\mathrm{CD}$ patients compared to controls. The top three features for classifying treatment response in the combined model were ko00633, the nitrotoluene degradation pathway, K07793, the putative tricarboxylic transport membrane protein, and Erysipelotrichi (the class containing the family Erysipelotrichaceae). Unlike for the combined disease model, MGS-derived functions were among the most highly ranked features (all six MGS functions are within the first eight top features).

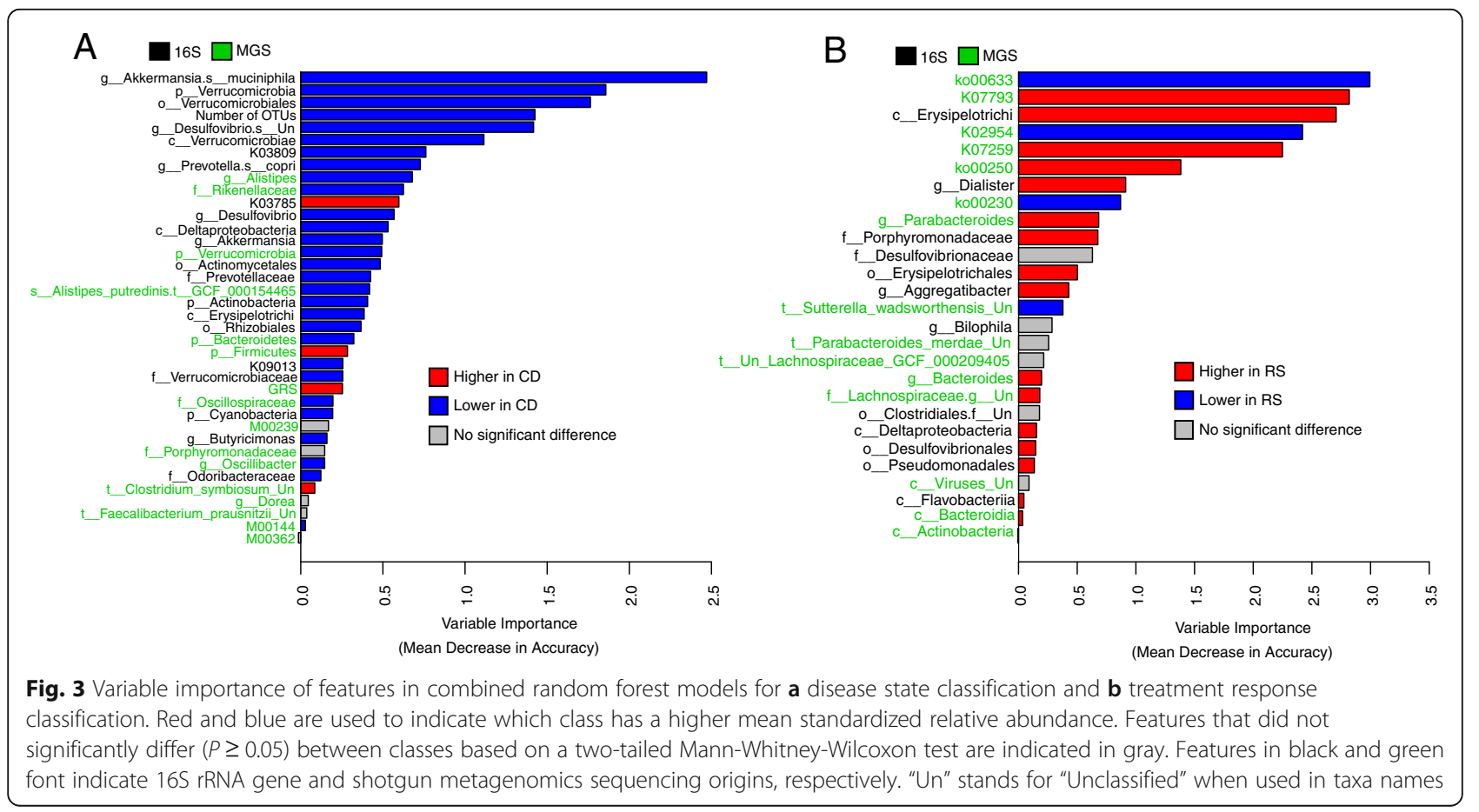




\section{Validating the best $16 \mathrm{~S}$ disease feature rankings in an independent cohort}

We validated the rankings of a subset of the $16 \mathrm{~S}$ features (excluding the unclassified species in Desulfovibrio) used in the combined model for disease state by training a new model based on these features on the RISK cohort [8], a large previously published dataset, that consisted of $16 \mathrm{~S}$ data for 731 biopsy samples (444 CD and 287 $\mathrm{CN}$ ) after processing. The goal of this analysis was to determine if the top features for classifying disease state would have similar relative importance ranks across both cohorts. Only $16 \mathrm{~S}$ sequencing of biopsy samples is available in this dataset and so we excluded GRS and the MGS features from this analysis. The new RF model based on this subset of features and trained on the RISK dataset was highly significant although less accurate than what we observed in our data (accuracy $=73.2 \%, P<0.001$ ). However, the relative ranking of these features was substantially different within the BISCUIT and RISK cohorts (Fig. 3a and Additional file 1: Figure S10). The top features in the RISK model were the class Erysipelotrichi, the phylum Actinobacteria, and the KO K09013. In addition, $8 / 2116 \mathrm{~S}$ features were not statistically different between $\mathrm{CD}$ and control patients (M-W-W test $P \geq 0.05)$. In particular, both Desulfovibrio and Akkermansia did not significantly differ between CD and control patients within the RISK cohort.

\section{Discussion}

In this study, we have classified treatment-naïve pediatric CD patients by both their disease state and treatment response with high accuracies with many different microbial datasets. Since these microbial profiles were taken from intestinal biopsy samples, the main challenge of this study was to identify true microbial markers above the background of human DNA in the MGS data. Although we could identify microbial markers by generating much higher sequencing depth than is usual, the interpretation of analyses of this data come with the caveat that important rare taxa may have been below the detection threshold. For instance, although the RF models based on the MGS datasets were less accurate classifiers of disease state, this likely was impacted by the fact that the most informative genera in the $16 \mathrm{~S}$ data were undetected in many MGS samples. This observation suggests that the discrepancy between the $16 \mathrm{~S}$ and MGS taxonomic classification accuracies could be partially due to a relatively greater taxonomic depth of $16 \mathrm{~S}$ sequencing, currently cost-prohibitive for MGS of biopsy samples, which enabled rarer taxa to be identified.

Since the $16 \mathrm{~S}$ data does not face these challenges, interpreting the analyses based on these datasets is more straightforward. Indeed, many of the top features in the significant $16 \mathrm{~S}$ datasets used to classify disease state (see
Additional file 4) have previously been associated with IBD. For instance, sulfur-reducing species within the Desulfovibrio genus have previously been positively linked to another form of IBD-ulcerative colitis [48], and Mottawea et al. recently showed the importance of hydrogen sulfide producers in colonic CD [49]. However, we found Desulfovibrio to be negatively associated with $\mathrm{CD}$ in our data, which could highlight a difference in microbiota between these two forms of IBD or merely reflect the different sampling strategies (stool, biopsy and MLI) between IBD studies to date. We also found Akkermansia muciniphila to have lower relative abundance in CD patients' biopsies, which has been previously observed [50]. The top 16S-inferred KOs are also related to functions previously associated with CD symptoms. The lower proportion of K09013 (Fe-S cluster assembly ATP-binding protein) in CD patients is interesting to find since intestinal inflammation in general has been associated with the breakdown of Fe-S clusters [51]. Similarly, both K03809 (tryptophan repressor binding protein) and K03785 (3-dehydroquinate dehydratase I), which is involved in tryptophan and other amino acid biosynthesis, in CD patients could be interesting markers since lower serum tryptophan levels has previously been associated with CD [52, 53]. However, in this analysis, these markers were both at higher levels in the unexpected direction (K03809 was lower in $\mathrm{CD}$ and K03785 was higher in CD).

The top MGS-identified features for classifying disease state also include several previously identified markers. The genus Alistipes is a known producer of short-chain fatty acids (SCFAs) [54]. This genus was at lower relative abundance in $\mathrm{CD}$ patients, which could be related to lower levels of certain SCFAs that have long been a hallmark of IBD [55-57]. In addition, although several key taxa identified by $16 \mathrm{~S}$ sequencing appeared to be below the detection threshold in the MGS samples, both Alistipes and Oscillibacter, which has previously been negatively associated with CD [58], were not identified in the $16 \mathrm{~S}$ data. The absence of these informative taxa is likely related to how certain lineages cannot be identified with high-resolution based on $16 \mathrm{~S}$ sequences. This difference highlights a trade-off in the MGS taxonomic results: improved taxonomic resolution at the cost of lower sensitivity, which has been discussed elsewhere [59]. The identification of the MGS-identified KEGG module M00144, which is involved in ATP synthesis, as being informative for classifying disease state is also interesting since IBD patients are known to have lower levels of intestinal ATP [60].

Similar to the RF models for disease state, many of the top features for classifying treatment response agreed with previous studies (see Additional file 5). For instance, the top $16 \mathrm{~S}$ genus, Dialister, was at higher 
abundance in RS patients, which is consistent with previous work [61]. Similarly, the bacterial family Erysipelotrichaceae has been linked to human health in several ways [62]. Although this taxon was not ranked highly, it is the only family within the top $16 \mathrm{~S}$-identified order, Erysipelotrichales, to pass pre-processing cut-offs. This order is found at higher relative abundance in RS patients. Erysipelotrichaceae are particularly of interest since they have been shown to decrease in abundance in $\mathrm{CD}$ patients given EEN therapy [63] and species within this family are positively linked to inflammation [64].

Several of the top MGS-identified KEGG functions also consistent with past work. The pathway ko00633, nitrotoluene degradation, has previously been identified as the most distinguishing pathway between EEN-treated CD patients and healthy controls [65]. Similarly, microbial glutathione and purine biosynthesis have previously been positively and negatively associated with Crohn's disease respectively [57]. In our dataset, the pathway ko00250, glutamate and other amino acid metabolism, was found at higher relative abundance in RS patients whereas ko00230, purine metabolism, was found at lower relative abundance in RS patients. In addition, both the genera Parabacteroides and Bacteroides have previously been found at higher abundance in CD patients at the time of surgical resection who remain in remission [66], which is the same direction we find here. Our previous work in this cohort determined that Sutterella wadsworthensis is unlikely to be involved in IBD pathogenesis [67]. However, since this species was one of the best predictive features for treatment response and was found at lower abundance in RS patients, it may still be clinically relevant. Although these results indicate that future $\mathrm{CD}$ treatments could be informed by the presence of these and other microbial markers, further work will be required to disentangle which markers are predictive of response to specific treatments.

The findings of Akkermansia muciniphila and the order and phylum (Verrucomicrobiales and Verrucomicrobia, respectively) that contain this species as the top three features for classifying disease state, highlights the importance of this taxon in our dataset. High levels of $A$. muciniphila in donor's stool has recently been found to be a strong predictor of remission in ulcerative colitis patients undergoing fecal microbiota transplantation treatment [68]. This finding taken together with our and others' observation of lower A. muciniphila abundance in CD patients suggests that this species is a useful biomarker for gut health. Similarly, the relative importance of alpha-diversity compared to genetic risk was also shown in this combined model. This finding illustrates the importance of microbial features in CD development, as compared with the weak contribution of genetic markers for $\mathrm{CD}$ development and the influence of the inherited variants on microbiome composition [69]. The top MGS-identified features largely performed worse than the 16S-identified functions in the combined RF model for classifying disease. One interesting exception is the genus Alistipes (and its corresponding family Rikenellaceae).

In the combined RF model for treatment response, it is notable that MGS-identified functions were the most informative features. This observation could indicate that major metabolic shifts in the microbiome could be more informative for predicting treatment response than the presence of particular taxa, which is consistent with past results indicating that functions shift more consistently than taxa in $\mathrm{CD}$ patients [57]. Interestingly, functions were only found to be more informative for classifying patients by treatment response, and not by disease state. However, it is possible that with higher sequencing depth, MGSidentified features may have been more informative. Note that patients' GRS were not significantly different between RS and NR samples, which is consistent with a recent study indicating that the genetic contributions to $C D$ susceptibility are largely independent from the genetic contributions to CD prognosis [70].

Ideally the combined RF model trained on the top features from our cohort would also have been tested on the validation cohort. However, due to technical differences across the studies, such as different sampling protocols and different $16 \mathrm{~S}$ variable regions sequenced, the same model cannot be implemented for both datasets. In addition, variation in pathophysiology due to geography as well as differential microbial profiles due to different distributions of patient age and sex across the cohorts could also result in differences in predictive markers across the two cohorts. This issue highlights that additional work in this area is needed to facilitate the comparison of microbiome datasets from different studies. Nonetheless, the independent validation cohort enabled the ranking of features within the combined model for disease state to be evaluated. The ranking of these features did differ in this cohort although the number of OTUs, Verrucomicrobiales, and Verrucomicrobia remain within the top six features (Additional file 1: Figure S10). However, the genera Desulfovibrio and Akkermansia were not significantly different between $\mathrm{CD}$ and control patients within the RISK samples, which highlights the issue of comparing predictive features across different cohorts. Unfortunately, we were unable to validate the ranking of the top features for classifying treatment response on an independent dataset since there is no paired $16 \mathrm{~S}$ and MGS dataset with adequate sample size available to our knowledge. 


\section{Conclusions}

Here, we have integrated human genetic data with $16 \mathrm{~S}$ and MGS intestinal biopsy data to classify CD patients by disease state and treatment response for the first time. We found genera identified from $16 \mathrm{~S}$ data to be the best classifiers of each outcome. One possible explanation for why $16 \mathrm{~S}$ data was found to have higher performance than the MGS data could be that it enables much higher read depth for taxonomic assignment. This increased depth allows rare taxa to be identified, which was the case for the top $16 \mathrm{~S}$-identified genera. The biological importance of rare taxa in $\mathrm{CD}$ pathogenesis warrants further consideration, and indeed, rarity may prove an important bias in culture-based studies of the IBD microbiome. Although we found alpha-diversity to be a clear marker for disease state, GRS was relatively less informative. This result is perhaps not surprising since microbial shifts are likely causally related to disease onset, although the direction is unclear. In contrast, GRS has been developed as a metric for assessing disease risk at any point in a patient's life, including well before onset, but has not been of great influence in predicting onset or treatment stratification [1]. The multi-genomics machine learning approach presented in this study could be extended in the future to other diseases and to other data types such as transcriptomics and metabolomics to better understand the relative importance of each of these features. These models will provide new insights into the multifactorial nature of $\mathrm{CD}$ and help highlight cohort-specific as well as fundamental contributors to disease pathophysiology and may result in novel signatures to predict and guide personalized treatments.

\section{Additional files}

Additional file 1: Figure S1. Stacked bar-chart showing the relative abundance of microbial classes across the metagenomic sequencing data. Figure S2. Stacked bar-chart showing the relative abundance of bacterial classes across the 165 rRNA gene sequencing data. Figure S3. Barplots comparing random forest model accuracies based on nonrarefied-centered log-ratio transformed and rarefied 165 rRNA gene taxa abundances. Figure S4. Boxplots of genetic risk scores and the number of observed OTUs, between Crohn's disease and control patients. Figure S5. Distribution of variable importance for $16 \mathrm{~S}$ rRNA gene-identified genera disease classification. Figure S6. Boxplots of the relative abundance of the top $16 \mathrm{~S}$ rRNA gene sequencing-identified genera for classifying disease state compared between sequencing technologies. Figure S7. Boxplots of genetic risk score and alpha-diversity between responders and non-responders to treatment. Figure S8. Pairwise Spearman correlation coefficients between features in the combined disease random forest. Figure S9. Pairwise Spearman correlation coefficients between features in the combined treatment response random forest. Figure S10. Features ranked by their relative importance for classifying disease state in the RISK validation cohort. Table S1. Table of demographic and phenotypic characteristics of pediatric patients. Table 2. Table of phenotypic characteristics and treatments of pediatric patients. (PDF $2350 \mathrm{~kb}$ )

Additional file 2: Sample sequencing coverage and metadata. (XLSX 49 kb)
Additional file 3: Random forest model summaries. (XLSX 65 kb)

Additional file 4: Ranking of variable importance in significant disease state models. (XLSX $226 \mathrm{~kb}$ )

Additional file 5: Ranking of variable importance in significant treatment response models. (XLSX $151 \mathrm{~kb})$

\section{Acknowledgements}

The authors would like to thank Dr. Hong Gu and Dr. Toby Kenney for helpful discussions. Dr. Mike Bisset, Dr. Sabari Loganathan, Dr. Gamal Mahdi, Dr. Dagmar Kastner-Cole, Dr. Andy Barclay, Dr. Jon Bishop, Dr. Diana Flynn, and Dr. Paraic McGrogan who all helped identify patients for BISCUIT and provided clinical samples during busy clinical diagnostic endoscopy lists.

\section{Funding}

BISCUIT was funded by a Clinical Academic Fellowship from the Chief Scientist Office (Scotland)—CAF/08/01. RH and RKR are supported by Career Researcher Fellowships from NHS Research Scotland.

The Glasgow Paediatric IBD team is supported by the Catherine McEwan Foundation. JVL was supported by a NASPGHAN/CCFA Young Investigator Development award (2013-2015), a Nova Scotia Health Research Foundation (NSHRF) establishment award (2015-2017), a Future Leaders in Inflammatory Bowel Disease (FLIBD) Program grant (2015-2016), a Dalhousie Medical Research Foundation equipment grant (2015-2016), a donation from the MacLeod family, an IWK Health Centre Research Associateship grant (Jessica Moore-Connors) and a Canadian Institutes of Health Research (CIHR)-CAG-CCC New Investigator Award (2015-2020: 201412XGP 340307-205026), a Canadian Foundation of Innovation John R. Evans Leadership fund (\#35235), and J. by a CIHR-SPOR-Chronic Diseases grant (Inflammation, Microbiome, and Alimentation: Gastro-Intestinal and Neuropsychiatric Effects: the IMAGINESPOR chronic disease network). JPB is supported by grants from CIHR (CMF-108026) and the Atlantic Computational Excellence Network (ACEnet 2011-2285). MGIL is supported by a Natural Sciences and Engineering Research Council Discovery Grant.

\section{Availability of data and materials}

All custom scripts used for this study are available at https://github.com/ LangilleLab/CD_RF_microbiome. The 165 rRNA gene and metagenomic sequencing data used in this study are available under accession PRJEB21933 at the European Nucleotide Archive.

\section{Authors' contributions}

GMD performed the statistical analyses and wrote the paper. $\mathrm{RH}$ designed the project, recruited all participants, collected raw data from patients, reviewed treatment response with $\mathrm{RT}$, and performed all DNA extraction. C $\mathrm{J}$ processed the $16 \mathrm{~S}$ rRNA gene sequencing data and called human variants. KD processed the metagenomic sequencing data and identified microbial taxa and functions. AMC produced the 165 rRNA gene sequencing data. JPB helped design the statistical analyses. RT collected patient metadata. EME was principal investigator for BISCUIT and is sample custodian for the study. RKR helped design the study. GLH, MGIL, and JVL supervised the project. All authors reviewed and agreed upon the final manuscript.

Ethics approval and consent to participate

Ethical approval was granted by North of Scotland Research Ethics Service (09/S0802/24), and written informed consent was obtained from the parents of all subjects. Informed assent was also obtained from older children who were deemed capable of understanding the nature of the study. This study is publicly registered on the United Kingdom Clinical Research Network Portfolio (9633). An ethics amendment allowed further review of the cohort participants' therapies and clinical response for the first year following diagnosis.

Consent for publication

Not applicable

Competing interests

The authors declare that they have no competing interests. 


\section{Publisher's Note}

Springer Nature remains neutral with regard to jurisdictional claims in published maps and institutional affiliations.

\begin{abstract}
Author details
'Department of Microbiology and Immunology, Dalhousie University, Halifax, NS, Canada. ${ }^{2}$ Department of Paediatric Gastroenterology, Royal Hospital for Children, Glasgow, UK. ${ }^{3}$ Department of Pharmacology, Dalhousie University, Halifax, NS, Canada. ${ }^{4}$ Department of Biology, Dalhousie University, Halifax, NS, Canada. ${ }^{5}$ CGEB-Integrated Microbiome Resource (IMR), Dalhousie University, Halifax, NS, Canada. 'Department of Medicine, St George and Sutherland Clinical School, UNSW, Sydney, NSW, Australia. 'Department of Pediatrics, Dalhousie University, Halifax, NS, Canada.
\end{abstract}

Received: 18 July 2017 Accepted: 2 January 2018

Published online: 15 January 2018

\section{References}

1. Cleynen I, Boucher G, Jostins L, Schumm LP, Zeissig S, Ahmad T, et al. Inherited determinants of Crohn's disease and ulcerative colitis phenotypes: a genetic association study. Lancet. 2016;387:156-67.

2. Neovius M, Arkema EV, Blomqvist P, Ekbom A, Smedby KE. Patients with ulcerative colitis miss more days of work than the general population, even following colectomy. Gastroenterology. 2013;144:536-43.

3. Cho JH. The genetics and immunopathogenesis of inflammatory bowel disease. Nat Rev Immunol. 2008;8:458-66.

4. Molodecky NA, Soon IS, Rabi DM, Ghali WA, Ferris M, Chernoff G, et al Increasing incidence and prevalence of the inflammatory bowel diseases with time, based on systematic review. Gastroenterology. 2012;142:46-54.

5. Henderson P, Hansen R, Cameron FL, Gerasimidis K, Rogers P, Bisset WM, et al. Rising incidence of pediatric inflammatory bowel disease in Scotland. Inflamm Bowel Dis. 2012;18:999-1005.

6. Ananthakrishnan AN. Epidemiology and risk factors for IBD. Nat Rev Gastroenterol Hepatol. 2015;12:205-17.

7. Seksik P, Rigottier-Gois L, Gramet G, Sutren M, Pochart P, Marteau P, et al. Alterations of the dominant faecal bacterial groups in patients with Crohn's disease of the colon. Gut. 2003:52:237-42.

8. Gevers D, Kugathasan S, Denson LA, Vázquez-Baeza Y, Van Treuren W, Ren $B$, et al. The treatment-naive microbiome in new-onset Crohn's disease. Cell Host Microbe. 2014;15:382-92.

9. Hansen R, Russell RK, Reiff C, Louis P, Mclntosh F, Berry SH, et al. Microbiota of de-novo pediatric IBD: increased Faecalibacterium prausnitzii and reduced bacterial diversity in Crohn's but not in ulcerative colitis. Am J Gastroenterol. 2012;107:1913-22.

10. Pascal V, Pozuelo M, Borruel N, Casellas F, Campos D, Santiago A, et al. A microbial signature for Crohn's disease. Gut. 2017;66:813-22.

11. Sokol H, Seksik P. The intestinal microbiota in inflammatory bowel diseases: time to connect with the host. Curr Opin Gastroenterol. 2010;26:327-31.

12. Shaw SY, Blanchard JF, Bernstein CN. Association between the use of antibiotics in the first year of life and pediatric inflammatory bowel disease. Am J Gastroenterol. 2010;105:2687-92.

13. McGovern DPB, Kugathasan S, Cho JH. Genetics of inflammatory bowel diseases. Gastroenterology. 2015;149:1163-76.

14. De Souza HSP, Fiocchi C. Immunopathogenesis of IBD: current state of the art. Nat Rev Gastroenterol Hepatol. 2016;13:13-27.

15. Jostins L, Ripke S, Weersma RK, Duerr RH, McGovern DP, Hui KY, et al. Hostmicrobe interactions have shaped the genetic architecture of inflammatory bowel disease. Nature. 2012:491:119-24.

16. Halme L, Paavola-Sakki P, Turunen U, Lappalainen M, Färkkilä M, Kontula K Family and twin studies in inflammatory bowel disease. World Gastroenterol. 2006;12:3668-72.

17. Hansen R, Berry SH, Mukhopadhya I, Thomson JM, Saunders KA, Nicholl CE, et al. The microaerophilic microbiota of de-novo paediatric inflammatory bowel disease: the BISCUIT Study. PLoS One. 2013;8:e58825.

18. Levine A, Griffiths A, Markowitz J, Wilson DC, Turner D, Russell RK, et al. Pediatric modification of the Montreal classification for inflammatory bowel disease: the Paris classification. Inflamm Bowel Dis. 2011;17:1314-21.

19. Langmead B, Salzberg SL. Fast gapped-read alignment with Bowtie 2. Nat Methods. 2012:9:357-9.
20. Segata N, Waldron L, Ballarini A, Narasimhan V, Jousson O, Huttenhower C Metagenomic microbial community profiling using unique clade-specific marker genes. Nat Methods. 2012;9:811-4.

21. Abubucker S, Segata N, Goll J, Schubert AM, Izard J, Cantarel BL, et al. Metabolic reconstruction for metagenomic data and its application to the human microbiome. PLoS Comput Biol. 2012;8:e1002358.

22. Manor O, Borenstein E. MUSiCC: a marker genes based framework for metagenomic normalization and accurate profiling of gene abundances in the microbiome. Genome Biol. 2015;16:53.

23. Li H, Durbin R. Fast and accurate short read alignment with BurrowsWheeler transform. Bioinformatics. 2009;25:1754-60.

24. McKenna A, Hanna M, Banks E, Sivachenko A, Cibulskis K, Kernytsky A, et al. The genome analysis toolkit: a MapReduce framework for analyzing next-generation DNA sequencing data. Genome Res. 2010;20: 1297-303.

25. DePristo MA, Banks E, Poplin R, Garimella KV, Maguire JR, Hartl C, et al. A framework for variation discovery and genotyping using next-generation DNA sequencing data. Nat Genet. 2011;43:491-8.

26. Van der Auwera GA, Carneiro MO, Hartl C, Poplin R, del Angel G, LevyMoonshine A, et al. From fastQ data to high-confidence variant calls: the genome analysis toolkit best practices pipeline. Curr Protoc Bioinformatics. 2013:43:1-33.

27. Danecek P, Auton A, Abecasis G, Albers CA, Banks E, DePristo MA, et al. The variant call format and VCFtools. Bioinformatics. 2011;27:2156-8.

28. Delaneau O, Marchini J, Zagury J-F. A linear complexity phasing method for thousands of genomes. Nat Methods. 2012;9:179-81.

29. Howie BN, Donnelly P, Marchini J. A flexible and accurate genotype imputation method for the next generation of genome-wide association studies. PLoS Genet. 2009;5:e1000529.

30. Howie B, Marchini J, Stephens M. Genotype imputation with thousands of genomes. G3 (Bethesda). 2011;1:457-70.

31. 1000 Genome Project Consortium. A global reference for human genetic variation. Nature. 2015;526:68-74.

32. Purcell $S$, Neale B, Todd-Brown $K$, Thomas L, Ferreira MAR, Bender D, et al. PLINK: a tool set for whole-genome association and population-based linkage analyses. Am J Hum Genet. 2007:81:559-75.

33. Jostins $L$, Levine AP, Barrett JC. Using genetic prediction from known complex disease loci to guide the design of next-generation sequencing experiments. PLoS One. 2013;8:e76328.

34. Liu JZ, van Sommeren S, Huang H, Ng SC, Alberts R, Takahashi A, et al. Association analyses identify 38 susceptibility loci for inflammatory bowel disease and highlight shared genetic risk across populations. Nat Genet. 2015:47:979-89.

35. Comeau AM, Douglas GM, Langille MG. Microbiome Helper: a custom and streamlined workflow for microbiome research. mSystems. 2017;2:e00127-16.

36. Zhang J, Kobert K, Flouri T, Stamatakis A. PEAR: a fast and accurate Illumina paired-end read merger. Bioinformatics. 2014;30:614-20.

37. Edgar RC, Haas BJ, Clemente JC, Quince C, Knight R. UCHIME improves sensitivity and speed of chimera detection. Bioinformatics. 2011;27:2194-200.

38. DeSantis TZ, Hugenholtz P, Larsen N, Rojas M, Brodie EL, Keller K, et al. Greengenes, a chimera-checked 16S rRNA gene database and workbench compatible with ARB. Appl Environ Microbiol. 2006;72:5069-72.

39. Kopylova E, Noé L, Touzet H. SortMeRNA: fast and accurate filtering of ribosomal RNAs in metatranscriptomic data. Bioinformatics. 2012:28:3211-7.

40. Langille MGI, Zaneveld J, Caporaso JG, McDonald D, Knights D, Reyes JA, et al. Predictive functional profiling of microbial communities using 165 rRNA marker gene sequences. Nat Biotechnol. 2013;31:814-21.

41. Gloor GB, Wu JR, Pawlowsky-Glahn V, Egozcue JJ. It's all relative: analyzing microbiome data as compositions. Ann Epidemiol. 2016;26:322-9.

42. Palarea-Albaladejo J, Martín-Fernández JA. zCompositions-R package for multivariate imputation of left-censored data under a compositional approach. Chemom Intell Lab Syst. 2015:143:85-96.

43. Bolger AM, Lohse M, Usadel B. Trimmomatic: a flexible trimmer for Illumina sequence data. Bioinformatics. 2014;30:2114-20.

44. Liaw A, Wiener M. Classification and regression by random forest. R News. 2002;2:18-22.

45. Murphy MA, Evans JS, Storfer A, Murphy MA, Evans JS, Storfer A. Quantifying Bufo boreas connectivity in Yellowstone National Park with landscape genetics. Ecology. 2016;91:252-61.

46. Kuhn M. Building predictive models in R using the caret package. J Stat Softw. 2008;28:1-26. 
47. Kanehisa M, Goto S. KEGG: Kyoto encyclopedia of genes and genomes. Nucleic Acids Res. 2000;28:27-30.

48. Rowan F, Docherty NG, Murphy M, Murphy B, Coffey JC, O'Connell PR. Desulfovibrio bacterial species are increased in ulcerative colitis. Dis Colon rectum. 2010;53:1530-6.

49. Mottawea W, Chiang CK, Mühlbauer M, Starr AE, Butcher J, Abujamel T, et al. Altered intestinal microbiota-host mitochondria crosstalk in new onset Crohn's disease. Nat Commun. 2016;7:13419.

50. Dunn KA, Moore-Connors J, Maclntyre B, Stadnyk AW, Thomas NA, Noble A, et al. Early changes in microbial community structure are associated with sustained remission after nutritional treatment of pediatric Crohn's disease. Inflamm Bowel Dis. 2016;22:2853-62.

51. Schumann S, Alpert C, Engst W, Loh G, Blaut M. Dextran sodium sulfateinduced inflammation alters the expression of proteins by intestinal Escherichia coli strains in a gnotobiotic mouse model. Appl Environ Microbiol. 2012;78:1513-22.

52. Gupta NK, Thaker Al, Kanuri N, Riehl TE, Rowley CW, Stenson WF, et al. Serum analysis of tryptophan catabolism pathway: correlation with Crohn's disease activity. Inflamm Bowel Dis. 2012;18:1214-20.

53. Nikolaus S, Schulte B, Al-Massad N, Thieme F, Schulte DM, Bethge J, et al. Increased tryptophan metabolism is associated with activity of inflammatory bowel diseases. Gastroenterology. 2017;153:1504-16.

54. Brown CT, Davis-Richardson AG, Giongo A, Gano KA, Crabb DB, Mukherjee N, et al. Gut microbiome metagenomics analysis suggests a functional model for the development of autoimmunity for type 1 diabetes. PLoS One. 2011;6:e25792.

55. Treem WT, Ahsan N, Shoup M, Hyams J. Fecal short-chain fatty acids in children with inflammatory bowel disease. J Pediatr Gastroenterol Nutr. 1994;18:159-64.

56. Huda-Faujan N, Abdulamir AS, Fatimah AB, Anas OM, Shuhaimi M, Yazid AM, et al. The impact of the level of the intestinal short chain fatty acids in inflammatory bowel disease patients versus healthy subjects. Open Biochem J. 2010;4:53-8.

57. Morgan XC, Tickle TL, Sokol H, Gevers D, Devaney KL, Ward DV, et al. Dysfunction of the intestinal microbiome in inflammatory bowel disease and treatment. Genome Biol. 2012;13:R79.

58. Mondot S, Kang S, Furet JP, Aguirre de Carcer D, McSweeney C, Morrison M, et al. Highlighting new phylogenetic specificities of Crohn's disease microbiota. Inflamm Bowel Dis. 2011;17:185-92.

59. Tessler M, Neumann JS, Afshinnekoo E, Pineda M, Hersch R, Velho LFM, et al. Large-scale differences in microbial biodiversity discovery between $16 \mathrm{~S}$ amplicon and shotgun sequencing. Sci Rep. 2017;7:6589.

60. Schürmann G, Brüwer M, Klotz A, Schmid KW, Senninger N, Zimmer K-P. Transepithelial transport processes at the intestinal mucosa in inflammatory bowel disease. Int J Color Dis. 1999;14:41-6.

61. Mondot S, Lepage P, Seksik P, Allez M, Tréton X, Bouhnik Y, et al. Structural robustness of the gut mucosal microbiota is associated with Crohn's disease remission after surgery. Gut. 2016;65:954-62.

62. Kaakoush NO. Insights into the role of Erysipelotrichaceae in the human host. Front Cell Infect Microbiol. 2015;5:84.

63. Kaakoush NO, Day AS, Leach ST, Lemberg DA, Nielsen S, Mitchell HM. Effect of exclusive enteral nutrition on the microbiota of children with newly diagnosed Crohn's disease. Clin Transl Gastroenterol. 2015;6:e71.

64. Dinh DM, Volpe GE, Duffalo C, Bhalchandra S, Tai AK, Kane AV, et al. Intestinal microbiota, microbial translocation, and systemic inflammation in chronic HIV infection. J Infect Dis. 2015;211:19-27.

65. Dunn KA, Moore-Connors J, Maclntyre B, Stadnyk A, Thomas NA, Noble A, et al. The gut microbiome of pediatric Crohn's disease patients differs from healthy controls in genes that can influence the balance between a healthy and dysregulated immune response. Inflamm Bowel Dis. 2016;22:2607-18.

66. De Cruz P, Kang S, Wagner J, Buckley M, Sim WH, Prideaux L, et al. Association between specific mucosa-associated microbiota in Crohn's disease at the time of resection and subsequent disease recurrence: a pilot study. J Gastroenterol Hepatol. 2015;30:268-78.

67. Mukhopadhya I, Hansen R, Nicholl CE, Alhaidan YA, Thomson JM, Berry SH, et al. A comprehensive evaluation of colonic mucosal isolates of sutterella wadsworthensis from inflammatory bowel disease. PLoS One. 2011;6:e27076.

68. Kump P, Wurm P, Gröchenig HP, Wenzl H, Petritsch W, Halwachs B, et al The taxonomic composition of the donor intestinal microbiota is a major factor influencing the efficacy of faecal microbiota transplantation in therapy refractory ulcerative colitis. Aliment Pharmacol Ther; 2017. Online: https://doi.org/10.1111/apt.14387.
69. Turpin W, Espin-Garcia O, Xu W, Silverberg MS, Kevans D, Smith Ml, et al. Association of host genome with intestinal microbial composition in a large healthy cohort. Nat Genet. 2016;48:1413-7.

70. Lee JC, Biasci D, Roberts R, Gearry RB, Mansfield JC, Ahmad T, et al. Genome-wide association study identifies distinct genetic contributions to prognosis and susceptibility in Crohn's disease. Nat Genet. 2017:49:262-8.

\section{Submit your next manuscript to BioMed Central and we will help you at every step:}

- We accept pre-submission inquiries

- Our selector tool helps you to find the most relevant journal

- We provide round the clock customer support

- Convenient online submission

- Thorough peer review

- Inclusion in PubMed and all major indexing services

- Maximum visibility for your research

Submit your manuscript at www.biomedcentral.com/submit
Biomed Central 